S. Mori

Nagoya Math. J.

Vol. 98 (1985), 43-66

\title{
ON 3-DIMENSIONAL TERMINAL SINGULARITIES
}

\author{
SHIGEFUMI MORI
}

\section{Introduction}

Canonical and terminal singularities are introduced by M. Reid [5], [6]. He proved that 3-dimensional terminal singularities are cyclic quotient of smooth points or $c D V$ points [6].

Let $(X, p)$ be a 3-dimensional terminal singularity of index $m$ with the associated $Z_{m}$-cover $(\tilde{X}, \tilde{p}) \rightarrow(X, p)$. If $(X, p)$ is a cyclic quotient singularity (i. e. if $(\tilde{X}, \tilde{p})$ is smooth), then it is known as Terminal Lemma (Danilov [3], D. Morrison-G. Stevens [4]) that there exist an integer $a$ prime to $m$ and coordinates $x, y, z$ of $(\tilde{X}, \tilde{p})$ which are $Z_{m}$-semi-invariants such that $\sigma(x)=\zeta x, \sigma(y)=\zeta^{-1} y, \sigma(x)=\zeta^{a} z$ for the standard generator $\sigma$ of $Z_{m}$, where $\zeta$ is a primitive $m$-th root of 1 . In this paper, we consider the case where $(\tilde{X}, \tilde{p})$ is a singular point and $m>1$. The main results are Theorems 12, 23, 25 and Remarks 12.2, 23.1, 25.1. These, together with the Terminal Lemma above, almost classify 3-dimensional terminal singularities.

Since $(\tilde{X}, \tilde{p})$ is an isolated singularity (or smooth) and is a hypersurface defined by a $Z_{m}$-semi-invariant power series (say $\varphi$ ), all deformations of $(X, p)$ are induced by deformations of $\varphi$ as a $Z_{m}$-semi-invariant power series $[2, \S \S 9-10]$. By Theorems 12, 23 and 25, one can see that there is a semi-invariant coordinate which has the same character as $\varphi$ (e.g. $z$ in Theorem 12, (1)), and hence every terminal singularity can be deformed to a cyclic quotient singularity (e.g. by $\varphi+\lambda z$ with parameter $\lambda$ for the case Theorem 12, (1)). This is not necessarily the case with canonical singularities.

The author expresses his hearty thanks to Professor S. Iitaka who allowed him to use his word processor, and to Professor M. Ishida for his helpful comments and pointing out mistakes in the original version.

As for the notation, we say that a monomial (say $u^{2}$ ) appears in a

Received December 21, 1983. 
power series (say $\varphi$ ) or $\varphi$ contains $u^{2}$ if $u^{2}$ appears with a non-zero coeffcient in the power series expansion of $\varphi$.

After having written up the paper, we learnt that Pinkham had proved similar (but slightly weaker) results (unpublished). We are grateful to Professor Kawamata, Pinkham, and Tsunoda for this information.

\section{$\S 1$. Criteria for terminal and canonical singularities}

Let $C$ be the field of complex numbers, and $C\{x\}$ denotes the ring of convergent power series in variables $x$.

Lemma 1. Let $(X, p)$ be the germ of an $n$-dimensional terminal (resp. canonical) singularity of index $m$. Let $\left(X^{\prime}, p^{\prime}\right)$ be the germ of an n-dimensional reduced Gorenstein variety and $f:\left(X^{\prime}, p^{\prime}\right) \rightarrow(X, p)$ a morphism such that $f$ factors as

$$
X^{\prime} \stackrel{g}{\longrightarrow} Y \stackrel{h}{\longrightarrow} X,
$$

where $h$ is a blow-up of $X$ and $g$ is quasi-finite. Let $\omega$ be a generator of $\omega_{X}^{(m)}$ at $p$. Then $f^{*} \omega$, as a meromorphic section of $\omega_{X^{\prime}}^{\otimes}$, vanishes (resp. is regular) along an arbitrary irreducible divisor $D \ni p$ such that $\operatorname{dim} f(D)$ $<n-1$.

Proof. Let $D$ be a divisor as in the lemma. Let $\pi: \tilde{X}^{\prime} \rightarrow X^{\prime}$ be the normalization, and $X^{\prime \prime} \subset \tilde{X}^{\prime}$ the complement of the singular locus of $\tilde{X}^{\prime}$. Since $\operatorname{codim}_{\tilde{X}^{\prime}}\left(\tilde{X}^{\prime}-X^{\prime \prime}\right) \geqq 2$ and since $\left.\left(\pi^{*} \omega_{X^{\prime}}\right)\right|_{X^{\prime \prime}} \supset \Omega_{X^{\prime \prime}}^{n}$, we may replace $\left(X^{\prime}, p^{\prime}\right)$ by $\left(X^{\prime \prime}, p^{\prime \prime}\right)$ for some smooth $p^{\prime \prime}$ such that $\pi\left(p^{\prime \prime}\right) \in D$. In other words, we may assume that $X^{\prime}$ is smooth. Hence in the factorization $X^{\prime} \rightarrow Y \rightarrow X$, we may assume that $Y$ is normal and $q=g(p)$ is a smooth point of $Y$ (by moving $p$ in $D$ if necessary). Then $h^{*} \omega$ vanishes (resp. is regular) along $g(D)$. Since $g:\left(X^{\prime}, p^{\prime}\right) \rightarrow(Y, q)$ is a morphism of manifolds, $f^{*} \omega=g^{*} h^{*} \omega$ vanishes (resp. is regular) along $D$. q.e.d.

Let $(X, p)$ be a 3-dimensional canonical singularity of index $m$ such that, for the associated $Z_{m}$-cover $\pi:\left(X^{\prime}, p^{\prime}\right) \rightarrow(X, p)[5,6],\left(X^{\prime}, p^{\prime}\right)$ is a hypersurface singularity. Then there exist $Z_{m}$-semi-invariants $x_{1}, \cdots, x_{4}$ in the analytic local ring $\mathcal{O}_{X^{\prime}, p^{\prime}}^{n}$ such that

$$
\begin{aligned}
& \rho\left(x_{i}\right)=\zeta^{c^{i}} x_{i}(i=1, \cdots, 4) \\
& \mathcal{O}_{X^{\prime}, p^{\prime}}^{h} \cong C\left\{x_{1}, \cdots, x_{4}\right\} /(\varphi),
\end{aligned}
$$


where $\rho$ (resp. $\zeta$ ) is a generator of $Z_{m}$ (resp. $\left.\mu_{m}\right), c=\left(c_{1}, \cdots, c_{4}\right) \in Z^{4}$ be such that $\operatorname{gcd}(c, m)=\operatorname{g.c.d} .\left\{c_{1}, \cdots, c_{4}, m\right\}=1$, and $\varphi$ is a semi-invariant. Since $\pi$ is unramified outside $p^{\prime}$, one has

$$
\left\{q \in X^{\prime} \mid x_{i}(q)=0 \quad \text { if } c_{i} \not \equiv 0(\bmod d)\right\}=\left\{p^{\prime}\right\}
$$

for any divisor $d>1$ of $m$. Hence, for any divisor $d>1$ of $m$, one has

$$
\operatorname{gcd}(c, m)=1, \quad \#\left\{i \in[1,4] \mid c_{i} \equiv 0(\bmod d)\right\} \leqq 1 .
$$

Now we reverse the process:

Notation (2.0). Let $Z_{n}$ act on $C\left\{x_{1}, \cdots, x_{4}\right\}$ by (1.1) with $\rho$ (resp. $\zeta$ ) a generator of $Z_{m}$ (resp. $\mu_{m}$ ), where $c \in Z^{4}$ satisfies (1.3) for an arbitrary divisor $d>1$ of $m$. Let $\varphi$ be a semi-invariant of $C\left\{x_{1}, \cdots, x_{4}\right\}$ such that $C\left\{x_{1}, \cdots, x_{4}\right\} /(\varphi)$ is normal, and let $\left(X^{\prime}, p^{\prime}\right)$ be the germ of a hypersurface at 0 defined by $(1.2)$, and $(X, p)=\left(X^{\prime}, p^{\prime}\right) / Z_{m}$.

Then we have

Theorem 2. Under Notation (2.0), let $\sigma$ be an arbitrary element of $Z_{m}$, and $a=\left(a_{1}, \cdots, a_{4}\right)$ a 4-ple of arbitrary integers $\geqq 0$ such that $\sigma\left(x_{i}\right)=\zeta^{a_{i}} x_{i}$ $(i=1, \cdots, 4)$ and that at least three of $a_{1}, \cdots, a_{4}$ are positive. Let $e(a)=$ $\max \left\{j \mid \varphi\left(x_{1} t^{a_{1}}, \cdots, x_{4} t^{a_{4}}\right) \equiv 0\left(\bmod t^{\jmath}\right)\right\} \quad$ and $|a|=a_{1}+\cdots+a_{4}$. Then if $(X, p)$ is terminal (resp. canonical), then $|a|-m-e(a)>0$ (resp. $\geqq 0)$.

Proof. (2.1) Let $X_{0}^{\prime}$ be $C^{4}$ with global coordinates $x_{1}, \cdots, x_{4}$, and let $Z_{m}$ act on $X_{0}^{\prime}$ by (1.1). Then $T^{\prime}=\left(C^{*}\right)^{4} \cap C^{4}=X_{0}^{\prime}$ is an affine torus embedding, and to the affine torus embedding $T^{\prime} \subset X_{0}^{\prime}$ correspond the group $\Gamma\left(T^{\prime}\right) \cong Z^{4}$ of 1 parameter subgroups of $T^{\prime}$ and a cone $C\left(X_{0}^{\prime}\right)$ of $\Gamma\left(T^{\prime}\right) \otimes_{Z} Q$. Let $\Gamma\left(T^{\prime}\right)=Z^{4}$ and $C\left(X_{0}^{\prime}\right)=Q_{+}^{4}$ in the standard way, where $\boldsymbol{Q}_{+}=\{q \in \boldsymbol{Q} \mid q \geqq 0\}$. Then, to $T=T^{\prime} \mid Z_{m} \rightarrow X_{0}=X_{0}^{\prime} / Z_{m}$, correspond $\Gamma(T)=$ $Z^{4}+Z c / m$ and $C\left(X_{0}\right)=Q_{+}^{4}$. By the definition of $a$, there exist integers $\beta, \gamma$ such that $\beta c_{i} \equiv \gamma a_{i} \bmod m$, where $\gamma$ is prime to $m$. Hence $a / m \in \Gamma(T)$.

(2.2) Let $\varphi^{\prime}$ be a convergent power series defined by

$$
\varphi^{\prime}\left(w, y_{1}, \cdots, y_{4}\right)=w^{-e(a)} \cdot \varphi\left(y_{1} w^{a_{1}}, \cdots, y_{4} w^{a_{4}}\right) .
$$

Then $\varphi^{\prime}(0, y)$ is a non-zero weighted homogeneous polynomial of weight $e(a)$ in $y_{1}, \cdots, y_{4}$ for weight $y_{i}=a_{i}$ (cf. (*) below). Since $y_{1} y_{2}$ and $y_{3} y_{4}$ are coprime, $\varphi^{\prime}(0, y)$ has a prime factor which is prime to $y_{1} y_{2}$ or $y_{3} y_{4}$. By symmetry, we may assume that $\varphi^{\prime}(0, y)$ has a prime factor prime to $y_{1} y_{2}$ and $a_{1}>0$. Since 
$(*)$

$$
\varphi^{\prime}\left(0, y_{1} t^{a_{1}}, \cdots, y_{4} t^{a_{4}}\right)=t^{e(a)} \varphi^{\prime}\left(0, y_{1}, \cdots, y_{4}\right)
$$

one can find $r_{2}, r_{3}, r_{4} \in C$ such that

$$
\varphi^{\prime}\left(0,1, r_{2}, r_{3}, r_{4}\right)=0 \text {. }
$$

(2.3) Let $e_{2}=(0,1,0,0), e_{3}=(0,0,1,0), e_{4}=(0,0,0,1) \in Z^{4}$, and let $C$ be the cone spanned by $a, e_{2}, e_{3}, e_{4}$ in $\boldsymbol{Q}^{4}$ and $M=Z e_{2} \oplus Z e_{3} \oplus Z e_{4} \subset \boldsymbol{Q}^{4}$. Then the commutative diagram

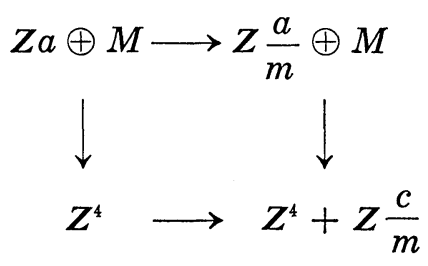

gives a commutative diagram of tori:

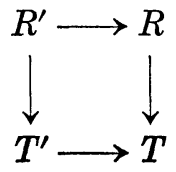

and $C \subset \Gamma\left(R^{\prime}\right) \oplus \boldsymbol{Q}$ gives a torus embedding

$$
R^{\prime} \subset Z_{0}^{\prime}=\operatorname{Spec} C\left[w, \bar{x}_{2}, \bar{x}_{3}, \bar{x}_{4}\right],
$$

where $a, e_{2}, e_{3}, e_{4}$ of $\Gamma\left(R^{\prime}\right)$ correspond to $w, \bar{x}_{2}, \bar{x}_{3}, \bar{x}_{4}$. Then

$$
R \subset Z_{0}=\operatorname{Spec} C\left[\bar{x}_{1}, \bar{x}_{2}, \bar{x}_{3}, \bar{x}_{4}\right],
$$

with $\bar{x}_{1}=w^{m}$, is the torus embedding corresponding to $C \subset \Gamma(R) \otimes \boldsymbol{Q}$, and commutative diagrams

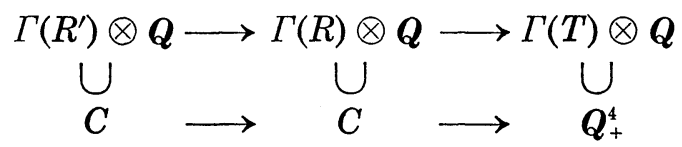

and

$$
\begin{aligned}
& \Gamma\left(R^{\prime}\right) \otimes \boldsymbol{Q} \longrightarrow \Gamma\left(T^{\prime}\right) \otimes \boldsymbol{Q} \longrightarrow \Gamma(T) \otimes \boldsymbol{Q} \\
& \stackrel{\cup}{C} \longrightarrow \cup_{Q_{+}^{4}} \longrightarrow \cup_{Q_{+}^{4}}^{\cup}
\end{aligned}
$$

give a commutative diagram 


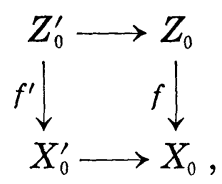

where $f^{\prime}$ is given by

$$
x_{1}=w^{a_{1}}, \quad x_{2}=\bar{x}_{2} w^{a_{2}}, \quad x_{3}=\bar{x}_{3} w^{a_{3}}, \quad x_{4}=\bar{x}_{4} w^{a_{4}} .
$$

If $T \subset Y_{0}$ is the torus embedding corresponding to $C \subset \Gamma(T) \otimes \boldsymbol{Q}$, then $Z_{0} \rightarrow Y_{0}$ is finite and $Y_{0} \rightarrow X_{0}$ is a blow-up.

(2.4) Let $s^{\prime}=V\left(w, \bar{x}_{2}-r_{2}, \bar{x}_{3}-r_{3}, \bar{x}_{4}-r_{4}\right) \in Z_{0}^{\prime}$ (resp. $s=$ the image of $s^{\prime}$ in $Z_{0}$ ), and let

$$
\psi\left(\bar{x}_{1}, \bar{x}_{2}, \bar{x}_{3}, \bar{x}_{4}\right)=w^{-e(a)} \cdot \varphi\left(w^{a_{1}}, \bar{x}_{2} w^{a_{2}}, \bar{x}_{3} w^{a_{3}}, \bar{x}_{4}^{a_{4}}\right),
$$

(note that the right hand side is a holomorphic function in $\bar{x}_{1}, \bar{x}_{2}, \bar{x}_{3}, \bar{x}_{4}$ defined near s.) Let $(Z, s) \subset\left(Z_{0}, s\right)$ (resp. $\left(Z^{\prime}, s^{\prime}\right) \subset\left(Z_{0}^{\prime}, s^{\prime}\right)$ ) be defined by $\psi=0$. Then $f$ (resp. $\left.f^{\prime}\right)$ induces $f:(Z, s) \rightarrow(X, p)$ (resp. $f^{\prime}:\left(Z^{\prime}, s^{\prime}\right) \rightarrow\left(X^{\prime}, p^{\prime}\right)$ ), which satisfies the conditions of Lemma 1 by (2.3). We have the following commutative diagram of natural morphisms:

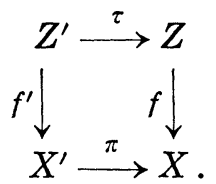

By Poincaré residue formula,

$$
\begin{gathered}
\omega_{X^{\prime}}=\operatorname{Res} \frac{d x_{1} \wedge d x_{2} \wedge d x_{3} \wedge d x_{4}}{\varphi}=-\frac{d x_{1} \wedge d x_{2} \wedge d x_{3}}{\varphi_{4}}, \\
f^{\prime *} \omega_{X^{\prime}}=-\frac{d\left(w^{a_{1}}\right) \wedge d\left(\bar{x}_{2} w^{a_{2}}\right) \wedge d\left(\bar{x}_{3} w^{a_{3}}\right)}{f^{\prime *} \varphi_{4}}
\end{gathered}
$$

where $\varphi_{4}=\partial \varphi / \partial x_{4}$. By calculation, one sees

$$
d\left(w^{a_{1}}\right) \wedge d\left(\bar{x}_{2} w^{a_{2}}\right) \wedge d\left(\bar{x}_{3} w^{a_{3}}\right)=a_{1} \cdot w^{a_{1}+a_{2}+a_{3}-1} \cdot d w \wedge d \bar{x}_{2} \wedge d \bar{x}_{3} .
$$

Since $f^{*} \varphi=w^{e(a)} \psi$, it follows from the chain rule that

$$
\left(f^{\prime *} \varphi_{4}\right) \cdot w^{a_{4}}=w^{e(a)} \psi_{4},
$$

where $\psi_{4}=\partial \psi / \partial \bar{x}_{4}$. Thus 


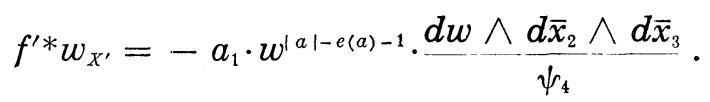

One also has

$$
\omega_{Z^{\prime}}=\operatorname{Res} \frac{d w \wedge d \bar{x}_{2} \wedge d \bar{x}_{3} \wedge d \bar{x}_{4}}{\psi}=-\frac{d w \wedge d \bar{x}_{2} \wedge d \bar{x}_{3}}{\psi_{4}} .
$$

One has

$$
f^{\prime *} \omega_{X^{\prime}}=a_{1} \cdot w^{|a|-e(a)-1} \omega_{Z^{\prime}}
$$

By construction, we have

$$
\tau^{*} \omega_{Z}=m \cdot w^{m-1} \omega_{Z^{\prime}} .
$$

Hence we have

$$
f^{*} \omega_{X^{\prime}}=\left(a_{1} / m\right) \cdot w^{|a|-e(a)-m} \tau^{*} \omega_{Z}
$$

Since $\pi: X^{\prime} \rightarrow X$ is unramified in codimension 1 by (1.3), one has $\pi^{*} \omega_{X}{ }^{(m)}=$ (unit) $\cdot \omega_{X^{\prime}}{ }^{\otimes m}$ near $p^{\prime}$ (by abuse of language, $\omega_{X}^{(m)}$ denotes one of its generators at $p$ ), and

$$
f^{*} \omega_{X}^{(m)}=(\text { unit }) \cdot \bar{x}_{1}^{|a|-e(a)-m} \omega_{Z}^{\otimes m} \quad \text { near } s .
$$

Since $\left\{\bar{x}_{1}=0\right\}$ is a divisor through $s$ collapsed by $f$, one has $|a|-e-m>0$ (resp. $\geqq 0$ ). q.e.d.

Under Notation (2.0), let $\chi_{1}, \cdots, \chi_{r} \in C\left\{x_{1}, \cdots, x_{4}\right\} \quad(r \geqq 2)$ be $Z_{m}$-semiinvariants with the same character such that $\chi_{i} C\left\{x_{1}, \cdots, x_{4}\right\}=u_{i} C\left\{x_{1}, \cdots, x_{4}\right\}$ for some monomial $u_{i}$ in $x_{1}, \cdots, x_{4}(i=1, \cdots, r)$ and that $u_{1}, \cdots, u_{r}$ are linearly independent over $C$ and the locus defined by $\chi_{1}=\cdots=\chi_{r}=0$ is of dimension $\leqq 1$. Let $\Phi$ be the linear system generated by $\chi_{1}, \cdots, \chi_{r}$, and assume that our $\varphi$ is written as

$$
\varphi=\sum_{i=1}^{r} \lambda_{i} \chi_{i}
$$

for some $\lambda=\left(\lambda_{1}, \cdots, \lambda_{r}\right) \in\left(C^{*}\right)^{r}$. By Bertini's theorem, $\varphi=0$ defines a normal variety for general $\lambda$ since the base locus of $\Phi$ is of dimension $\leqq 1$. Let $\sigma, a, \zeta$ be as in Theorem 2 , then the value of $e(a)$ given in Theorem 2 does not depend on the choice of $\lambda \in\left(C^{*}\right)^{r}$. Then under the notation of Theorem 2, the following is the corollary to the proof of Theorem 2. 


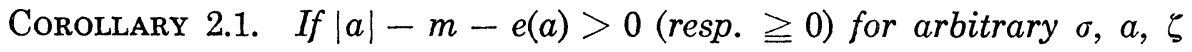
as in Theorem 2, then $(X, p)$ is terminal (resp. canonical) for general $\lambda$. If $(X, p)$ has an isolated singular point at $p$ (resp. canonical singularity outside $p$ ) for general $\lambda$, then one can add the extra conditions $a_{1}, \cdots, a_{4}>0$ on $a$ in the statement above.

Proof. Let $X_{0}=C^{4} / Z_{m}$ with respect to the action given above. Then $\Phi$ induces a linear system $\Phi_{0}$ (of Weil divisors) in a neighborhood of 0 in $X_{0}$ which is free from fixed components. By the conditions on $\chi_{i}$ 's, there exists a toric resolution $h: U_{0} \rightarrow X_{0}$ such that the proper transform $\Psi_{0}$ of $\Phi_{0}$ to $U_{0}$ is free from base points (principalizer of the coherent sheaf associated to $\Phi_{0}$ ). Then a general member $X$ of $\Phi_{0}$ is normal at 0 and its proper transform $U=h^{-1}[X]$ is smooth in a neighborhood of $h^{-1}(0)$. Thus it is enough to show that $h^{*} \omega_{X}^{(m)}$, as a meromorphic section of $\omega_{U}^{\otimes m}$, vanishes (resp. is regular) along $D_{0} \cap U$, where $D_{0} \subset U_{0}$ is an arbitrary exceptional divisor of $h$. We now use the notation of the proof of Theorem 2. Let $L_{+}$be the 1-simplex $\subset \boldsymbol{Q}_{+}^{4} \subset \Gamma(T) \otimes \boldsymbol{Q}$ corresponding to $D_{0}$. Let $a=\left(a_{1}, \cdots, a_{4}\right)$ be a 4-ple of integers $\geqq 0$ such that $Z a / m=$ $\boldsymbol{Q} L_{+} \cap \Gamma(T)$. By (1.3), the singular locus of $X_{0}$ is of dimension $\leqq 1$, and the base locus of $\Phi_{0}$ is of dimension $\leqq 1$. Thus one may assume that the image of $D_{0}$ to $X_{0}$ is of dimension $\leqq 1$, whence at least 3 of $a_{1}, \cdots, a_{4}$ are positive. Then the notation of (2.3) can be used, and the torus embedding $T \subset V_{0}=T \cup D_{0}$ corresponds to $L_{+} \subset \Gamma(T) \otimes \boldsymbol{Q} . \quad L_{+} \subset \Gamma(R) \otimes \boldsymbol{Q}$ corresponds to the open subset $W_{0}$ of $Z_{0}$ defined by $\bar{x}_{3} \bar{x}_{4} \neq 0$. Since $Z a / m=$ $\boldsymbol{Q} a / m \cap \Gamma(T),\left\{\bar{x}_{1}=0\right\}$ is not in the branch locus of $W_{0} \rightarrow V_{0}$. Let $V, W$ be the proper transforms of $X$ to $V_{0}$ and $W_{0}$, respectively. Then, since $\Psi_{0}$ is free from base points, $g: W \rightarrow V$ is unramified over general points of arbitrary irreducible components of $V \cap D_{0}$. Thus by

$$
g^{*} h^{*} \omega_{X}^{(m)}=(\text { unit }) \cdot \bar{x}_{1}^{|a|-e(a)-m} \omega_{w}^{\otimes m} \text { along } W \cap D_{0},
$$

(2.4), we have Corollary 2.1.

q.e.d.

COROLLARY 2.2. Under the assumptions of Theorem 2, assume that $m$ is odd, and

$$
\varphi=x_{1}^{2}+f\left(x_{2}, x_{3}, x_{4}\right) \quad\left(f \in C\left\{x_{2}, x_{s}, x_{4}\right\}\right) .
$$

Let $n=\max \left\{j \mid \varphi\left(x_{2} t^{a_{2}}, x_{3} t^{a_{3}}, x_{4} t^{a_{4}}\right) \equiv 0\left(t^{j}\right)\right\}$. Then

$$
\left.a_{2}+a_{3}+a_{4}>\text { (resp. } \geqq\right) \begin{cases}m+n / 2 & \text { if } n \text { is even } \\ m / 2+n / 2 & \text { if } n \text { is odd } .\end{cases}
$$


Proof. One has $2 \cdot w t x_{1} \equiv n(\bmod m)$. If $n$ is even, then we choose $a_{1}=n / 2$, keeping $a_{2}, a_{3}, a_{4}$ the same. Then $n / 2+a_{2}+a_{3}+a_{4}>$ (resp. $\left.\geqq\right)$ $m+n$. If $n$ is odd, then we choose $a_{1}=(m+n) / 2$, keeping $a_{2}, a_{3}, a_{4}$ the same. Then $(n+m) / 2+a_{2}+a_{3}+a_{4}>$ (resp. $\left.\geqq\right) m+n$. q.e.d.

For the approximation of $\varphi$, we need the standard:

Theorem 3. Let $\varphi \in C\{x\}$, where $x=\left(x_{1}, x_{2}, x_{3}, x_{4}\right)$. Assume that $\varphi$ has an isolated singular point at (0), and that $Z_{m}$ acts on $C\{x\}$ in such a way that $x_{1}, x_{2}, x_{3}, x_{4}$, and $\varphi$ are semi-invariants. Then for an arbitrary integer $b>0$, there exists an integer $n>0$ such that, for an arbitrary semiinvariant $\psi \in C\{x\}$ with the property $\psi \equiv \varphi(x)^{n}$, there exists an analytic $C$-automorphism $\sigma$ of $\boldsymbol{C}\{x\}$ commuting with $Z_{m}$-action (will be called a $Z_{m}$-automorphism, for short) such that $\sigma(\varphi)=\psi C, \sigma \equiv$ id modulo $(x)^{b}$.

This can be proved by applying the argument of [1, Lemma (5.11)] to the equation $\varphi(y)-\psi(x)=0$ in unknown variables $y=\left(y_{1}, y_{2}, y_{3}, y_{4}\right)$ with approximate solution $y^{0}=x$, where $m=1$ and $N=4$.

COROLlary 4. Under the notation and the assumptions of Theorem 3, if $\varphi \in(x)^{2}$ and if $x_{1} x_{2}$ appears in $\varphi$ and

$$
\left(\hat{\sigma}^{2} \varphi / \partial x_{1}^{2}(0)\right)\left(\partial^{2} \varphi / \partial x_{2}^{2}(0)\right)-\left(\partial^{2} \varphi / \partial x_{1} \partial x_{2}(0)\right)^{2} \neq 0,
$$

then there exist a $Z_{m}$-automorphism $\sigma$ of $C\{x\}$ such that

$$
\begin{aligned}
& \sigma\left(x_{3}\right)-x_{3}, \quad \sigma\left(x_{4}\right)-x_{4} \in(x)^{2}, \\
& \sigma(\varphi)=x_{1} x_{2}+f\left(x_{3}, x_{4}\right) \quad \text { for some } f \in C\left\{x_{3}, x_{4}\right\} .
\end{aligned}
$$

\section{§2. Notation and terminal singularities of type $c A$}

Assumption 5. Let $\varphi$ be an element of $(x, y, z, u)^{2} C\{x, y, z, u\}$ which has a $Z_{m}$-action $(m>1)$ such that $x, y, z, u, \varphi$ are semi-invariants. Assume that $\varphi$ has an isolated $c D V$ singularity at the origin (0), that the quotient of $\{\varphi=0\}$ by $Z_{m}$ has a terminal singularity at (0), and that the action of $Z_{m}$ is free on $U-(0)$, where $U \ni(0)$ is an open set of $\{\varphi=0\}$. By a $Z_{m}$-automorphism, we mean an analytic $C$-automorphism of $C\{x, y, z, u\}$ commuting with $Z_{m}$-action unless otherwise mentioned. We will keep these assumptions and notation, unless otherwise mentioned.

Notation 6. Fixing a primitive $m$-th root $\zeta$ of 1 , and given the $Z_{m}$-action above, we associate to each $\sigma \in Z_{m}$ a weight modulo $m$ (denoted 
by $\sigma-w t \bmod m) ; \sigma-w t(x) \equiv \mathrm{a}(\sigma), \cdots, \sigma-w t(u) \equiv d(\sigma)(\bmod m)$ are determined by $\sigma(x)=\zeta^{a(\sigma)} \cdot x, \cdots, \sigma(u)=\zeta^{d(\sigma)} \cdot u$. If $\sigma$ is a generator of $Z_{m}$, we may simply call $\sigma-w t$ a $w t$, if there is no danger of confusion. Order $v$ associates numbers $a, b, c, d>0$ to $x, y, z, u$ such that $a \equiv \sigma-w t(x), \cdots$, $d \equiv \sigma-w t(u) \bmod m$ for some $\sigma \in Z_{m}$. Then the order of $f$, or $v(f)$ is, by definition, $\max \left\{n \mid f\left(x t^{a}, y t^{b}, z t^{c}, u t^{d}\right) \equiv 0\left(t^{n}\right)\right\}$. We write $v \equiv \sigma-w t(\bmod m)$ if $v(x) \equiv w t x, \cdots, v(u) \equiv w t u(\bmod m)$. For two orders $v$ and $v^{\prime}$, we write $v \equiv v^{\prime}(\bmod m)$ if $v(x) \equiv v^{\prime}(x), \cdots, v(u) \equiv v^{\prime}(u)(\bmod m)$. For a positive integer $a$ and a number $b$ (or, $b \in R / a Z$ ), $(b)_{a}$ denotes the number $c$ such that $c-b \in a Z$ and $0<c \leqq a$. We define order $v=(\sigma-w t)_{m}$ by $v(x)=$ $(\sigma-w t x)_{m}, \cdots, v(u)=(\sigma-w t u)_{m}$.

Remark 7. Assumption 5 implies:

(1) if $e=(m, w t x)>1$, then some power of $x$ appears in $\varphi$ and $w t \varphi \equiv 0(\bmod e)$. Similar assertion holds also for $y, z, u$. (Since the action on $x$-axis is not free, $\{\varphi=0\}$ does not contain $x$-axis.)

(2) $(m, w t x, w t y)=1$. Similar assertion holds also for any other two distinct coordinate functions. (Otherwise the action is not free on $\{\varphi=0\} \cap x y$-plane which has dimension $>0$ at $(0))$.

THEOREM 8. If $x y$ appears in $\varphi$ and

$$
\left(\partial^{2} \varphi / \partial x^{2}(0)\right)\left(\partial^{2} \varphi / \partial y^{2}(0)\right)-\left(\partial^{2} \varphi / \partial x \partial y(0)\right)^{2} \neq 0,
$$

then one of the following holds (after exchanging $z, u$ if necessary):

(1) $w t x+w t y \equiv w t z \equiv 0 \bmod m$, and $w t u$, wt $x$, wt $y$ are prime to $m$.

(2) $m=4$ and there exists a generator $\sigma$ of $Z_{4}$ such that $\sigma$-wts of $x, y, z, u$ are $1,1,2,3 \bmod 4$ (see Supplement 8.1).

Proof. By Corollary 4, we may assume $\varphi=x y+f(z, u)$, where $f \in C$ $\{z, u\}$. Let $e=(w t x+w t y)_{m}, p=(e, m)$.

(8.1) Claim: One of $w t z$, wt $u$ is a multiple of $p$, and the other is prime to $m$. wt $x, w t y$ are prime to $m$.

Let $w t$ be $\sigma-w t$. Assume that $\sigma-w t z, \sigma-w t u \not \equiv 0(p)$ (and hence $p>1$ ). If necessary, we will replace $\sigma$ by $-\sigma$ to get

$$
(\sigma-w t z)_{p}+(\sigma-w t u)_{p} \leqq p .
$$

By Remark 7, (2), and

$$
\sigma-w t x+\sigma-w t y \equiv 0(p)
$$


one sees that $\sigma-w t x$ and $\sigma-w t y$ are prime to $p$. Hence

$$
(\sigma-w t x)_{p}+(\sigma-w t y)_{p}=p .
$$

Let $v$ be the order $(m / p) \cdot(\sigma-w t)_{p}=((m / p) \cdot \sigma-w t)_{m}$. Then $v(\varphi)=m$ and $v(x)+v(y)=m, v(z)+v(u) \leqq m$. Thus $v(x)+v(y)+v(z)+v(u) \leqq m+v(\varphi)$, which is a contradiction to Theorem 2, and hence $\sigma-w t z \equiv 0$ or $\sigma-w t u$ $\equiv 0(p)$. By symmetry of $z, u$, we may assume that $\sigma-w t z \equiv 0(p)$. Let $n=(\sigma-w t u, m)$. We will show that $n=1$. If $n>1$ then by Remark 7 , (1), $w t \varphi \equiv 0(n)$. Thus $n \mid p$ and $w t z \equiv w t u \equiv 0(n)$. This contradicts Remark 7, (2). Thus $n=1$. By Remark 7, (1), (2), if $n=(m, \sigma-w t x)>1$, then $w t x+w t y \equiv w t \varphi \equiv 0(n)$ and $n=(m, \sigma-w t x, \sigma-w t y)$. Thus $n=1$ by Remark 7, (2). Similar argument shows $(m, \sigma-w t y)=1$. This proves (8.1).

(8.2) Claim: By symmetry of $z$, $u$, we may assume that $w t z \equiv 0(p)$ and $w t u$ is prime to $m$. Then $p=(w t z, m)$, and $f(z, u)=g\left(z, u^{p}\right)$ for some convergent power series $g$.

Let $n=(w t z, m)$. Then $p \mid n$. By Remark 7, (1), one has $w t x+$ wt $y=0(n)$, whence $n \mid p$ and $n=p$.

(8.3) Claim: If $p<m$ and $\rho \in Z_{m}$ satisfies

$$
\rho-w t x+\rho-w t y \equiv \pm p(m)
$$

then $\rho$-wt $x, \rho$-wt $y, \rho$-wt $z, \rho-w t u \neq 0(m)$.

If $\rho-w t \equiv i \cdot w t(m)$, then $\mathrm{i} \cdot \mathrm{e} \equiv \pm p(m)$. Thus $i \neq \equiv(m)$, whence $i \cdot w t x, i \cdot w t y, i \cdot w t u \neq 0(m)$ by (8.1), (8.2). One also sees that $i$ is prime to $m / p>1$. Thus by (8.2), $\rho-w t z \equiv i \cdot(w t z) \not \equiv 0(m)$.

(8.4) Claim: Assume $p<m$. The number of elements $\rho \in Z_{m}$ such that

$$
\begin{gathered}
\rho-w t x+\rho-w t y \equiv \pm p(m), \quad \text { and } \\
(\rho-w t z)_{m}+(\rho-w t u)_{m} \leqq m
\end{gathered}
$$

is $\geqq p$ if $m>2 p$, and $\geqq p / 2$ if $m=2 p$.

As in (8.3), the number of $\rho$ 's with $(*)$ is the number of solutions $i$ $(0 \leqq i<m)$ for $i \cdot e \equiv \pm p(m)$, which is $2 p$ if $m>2 p, p$ if $m=2 p$. Now involution $\iota: \rho \rightarrow-\rho$ acts on $\Sigma=\{\rho$ with $(*)\}$ and $\rho$ or $-\rho$ satisfies (**) by (8.3). Thus (8.4) is settled. 
(8.5) If $p=m$, then (1) holds. We may now assume $p<m$, and let $\rho$ be an arbitrary element with $(*),(* *)$. Let $v=(\rho-w t)_{m}$, then by (8.3),

$$
\begin{aligned}
& v(x)+v(y)=p, m \pm p, \text { or } 2 m-p, \\
& v(z)+v(u) \leqq m .
\end{aligned}
$$

By Theorem 2,

$$
m+v(x)+v(y) \geqq v(x)+v(y)+v(z)+v(u)>m+v(\varphi),
$$

i.e. $v(\varphi)<v(x)+v(y)$. Thus there are two cases: (8.5.1) $v(\varphi)=p, v(x)+$ $v(y)=m+p$, and (8.5.2) $v(\varphi)=m-p, v(x)+v(y)=2 m-p$.

(8.5.1) Assume that $v(\varphi)=p$. Then $v\left(g\left(z, u^{p}\right)\right)=p$ by (8.2). Since $g \in(z, u)^{2}$ and $v(z) \geqq p(8.2)$, one has $p=v\left(g\left(z, u^{p}\right)\right)=p \cdot v(u)$ and $p \geqq 2$, hence $v(u)=1$ and $\rho-w t u \equiv 1(m)$. Thus there is at most one such $\rho$.

(8.5.2) Assume that $m>2 p$ and $v(\varphi)=m-p$. Then $v(x)=m-j$ and $v(y)=m-p+j(j=1, \cdots, p-1)$ by (8.3). Since $(\rho-w t x, \rho$-wt $y, m)$ $=1$, there are at most $p-1$ such $p$ 's.

(8.6) If $m=2 p$, then by (8.4) and (8.5.1), one sees that $m=2 p=4$. Then by choosing $w t$, one has $w t$ s of $x, y, z, u$ equal to $1,1,2, \alpha(\alpha=1,3)$. One sees $\alpha=3$ by applying Theorem 2. Whence one gets (2).

We now assume that $m>2 p$ and will derive a contradiction to finish the proof. Then by (8.4), (8.5.1) and (8.5.2), one sees that $p \geqq 2$ and there are exactly one $\rho$ in case (8.5.1) and exactly $p-1 \rho$ 's in case (8.5.2). We claim that $p=2$. If $p>2$, then let $\rho_{i}(i=1,2)$ in case (8.5.2) be such that $\rho_{i}-w t x \equiv m-i(i=1,2)$. Then $\rho_{2}=2 \rho_{1}$ and whence $-p \equiv \rho_{2}-w t \varphi$ $\equiv 2\left(\rho_{1}-w t \varphi\right) \equiv-2 p(m)$. This means that $p \equiv 0(m)$ and $m=p$, which contradicts $m>2 p$. Thus our claim that $p=2$ is proved. Let $\rho_{1} \in Z_{m}$ be in case (8.5.1) and $\rho_{2} \in Z_{m}$ in case (8.5.2). One has $\rho_{2}-w t x \equiv \rho_{2}-w t y \equiv m$ $-1(m)$ and $\rho_{2}$ is a generator of $Z_{m}$. Thus by $\left(\rho_{1}-w t x\right)_{m}+\left(\rho_{1}-w t y\right)_{m}=m$ +2 , one has $\rho_{1}-w t x \equiv \rho_{1}-w t y \equiv m / 2+1(\bmod m)$. Hence $\rho_{1}=(m / 2-1) \rho_{2}$. Let $\rho_{3}=2 \rho_{1}$. Then $\rho_{3}=-2 \rho_{2}$, and $\rho_{3}-w t$ s of $x, y, u$ are $2,2,2 \bmod m$. Let $w=\left(\rho_{3}-w t\right)_{m}$. Then by $m \equiv 0(2)$ and $m>2 p=4$, one has $m \geqq 6$. Thus $w(\varphi)=w(x)+w(y)=4$. Applying Theorem 2 to $w$, one has $w(z)>$ $m-2$. Hence $w(z)=m$ because $m$ is even and $\rho_{3} \equiv 0(2)$. Since $\rho_{3}=-$ $2 \rho_{2}$, one has $\rho_{2}-w t z \equiv 0$ or $m / 2$. Since $\rho_{2}$ is a generator of $Z_{m}$, one has $p=\left(\rho_{2}-w t z, m\right)=m$ or $m / 2$ by (8.2). Thus $m=p$, or $2 p$ ocntradicting our assumption $m>2 p$.

q.e.d. 
Supplement 8.1. In case (2), one sees that $g\left(z, u^{2}\right)$ contains $u^{2}$ in (8.5). Thus one sees: in case (2) of Theorem 8, modulo $Z_{4}$-automorphism, one has

$$
\varphi=x y+z^{n}+u^{2} \quad(n: o d d \geqq 3),
$$

where wts of $x, y, z, u$ are $1,1,2,3(\bmod 4)$.

Proof. By $Z_{4}$-automorphism: $z \rightarrow$ (const) $z, u \rightarrow($ const $) \cdot u$, keeping $x$ and $y$, one has $g=u^{2}\left(1+\alpha\left(z, u^{2}\right)\right)+z^{n}(1+\beta(z))$, where $\alpha \in(z, u), \beta \in(z)$, $n>1$. Since $\alpha, \beta$ are $Z_{4}$-invariant, one can change $u \cdot\left\{1+\alpha\left(z, u^{2}\right)\right\}^{(1 / 2)} \rightarrow$ $u, z \cdot\{1+\beta(z)\}^{(1 / n)} \rightarrow z$, to get $g=u^{2}+z^{n}$. $\quad$ q.e.d.

Lemma 9. If $x^{2}$ and $y^{2}$ appear in $\varphi$ and $\varphi_{2} \in k x^{2}+k y^{2}+k z^{2}+k u^{2}$, then $m=2,4$.

Proof. (9.1) Claim: $m$ is a power of 2 .

If $m$ is not a power of 2, we may assume that $m$ is odd and $>1$ by replacing $m$ by its odd factor. Then $w t x \equiv w t y$, and hence by $Z_{m}$-automorphism: $x \rightarrow x+\alpha \cdot y$, keeping $y, z$, $u$, we may assume that $\varphi$ contains $x y, x^{2}, y^{2}$. Thus by Theorem 8 , wt $x+w t y \equiv 0(m)$, wt $x$ and wt $y$ are prime to $\mathrm{m}$, which contradicts $w t x \equiv w t y$. Thus $m=1$, and hence $m$ is proved to be a power of 2 .

(9.2) Claim: If $m=8$, then degree 2 part $\varphi_{2}$ of $\varphi$ is a quadratic form of rank 3 .

By $2 \cdot w t x \equiv 2 \cdot w t y(8)$, one has $w t x \equiv w t y(4)$. Thus by $Z_{4}$-automorphism: $x \rightarrow x+\alpha y$ keeping $y, z, u$, one may assume $x^{2}, y^{2}, x y$ appear in $\varphi$ and $m=4$. Then by Theorem 8 and Supplement $8.1, \varphi_{2}$ has rank 3 .

(9.3) Claim: $m \mid 4$.

If $8 \mid m$ can occur, the case $m=8$ occurs. if $m=8$, then $\varphi_{2}$ contains $x^{2}, y^{2}, z^{2}$ (by exchanging $z, u$ if necessary.) By $2 w t x \equiv 2 w t y \equiv 2 w t z$ (8), one sees that two of $w t x, w t y, w t z$ are congruent mod 8. We may assume $w t x \equiv w t y(8)$, without loss of generality. By $Z_{8}$-automorphism: $x \rightarrow x+$ $\alpha y$, keeping $y, z, u$, one may assume that $\varphi$ contains $x^{2}, y^{2}, x y$ for $m=8$. This contradicts Theorem 8. Thus Lemma 9 is proved.

Lemma 10. If $x^{2}$ and $y^{2}$ appear in $\varphi$ and $\varphi \in k x^{2}+k y^{2}+k z^{2}+k u^{2}$ and $m=4$, then

(1) if $w t x \equiv w t y \bmod 4$, then after exchanging $z$, $u$ (if necessary) and choosing a generator $\sigma$ of $Z_{4}$, one has $\sigma$-wts of $x, y, z$, u equal to $1,1,2,3$ $\bmod 4$, and modulo $Z_{4}$-automorphism, 


$$
\varphi=x^{2}+y^{2}+z^{n}+u^{2}(n: \text { odd } \geqq 3), \quad \text { and }
$$

(2) if wt $x \not \equiv w t y(4)$, then after exchanging $z, u$ and exchanging $x, y$ (if necessary) and choosing a generator $\sigma$ of $Z_{4}$, one has $\sigma$-wts of $x, y, z, u$ equal to $1,3,2,1 \bmod 4$.

Proof. Case (1) is due to Supplement 8.1 (cf. argument of Lemma 9).

Case (2): By $2 w t x \equiv 2 w t y(4)$, one sees that $w t x$, wty are odd by Remark 7, (2). Hence $w t \varphi \equiv 2$ (4). Applying Theorem 8 to $m=2$, wt $z$ or $w t u$ is even (cf. argument of Lemma 9). Let us assume that $w t z$ is even. By Remark 7, (1), $\varphi$ contains some power of $z$, whence $w t z \equiv 2$ (4). Changing $\sigma$ to $-\sigma$ if necessary, one gets (2).

LEMma 11. If $x^{2}$ and $y^{2}$ appear in $\varphi$ and $\varphi \in k x^{2}+k y^{2}+k z^{2}+k u^{2}$ and $m=2$, then

(1) if wt $x \equiv w t y \bmod 2$, then after exchanging $z$, $u$ (if necessary), one has wts of $x, y, z, u$ equal to $1,1,0,1 \bmod 2$, and modulo $Z_{2}$-automorphism, $\varphi=x^{2}+y^{2}+f\left(z, u^{2}\right)$ for some $f \in C\{z, u\}$,

(2) if $w t x \not \equiv w t y \bmod 2$, then after exchanging $x, y$ (if necessary), one has wts of $x, y, z$, $u$ equal to $1,0,1,1 \bmod 2$, and modulo $Z_{2}$-automorphism, $\varphi=x^{2}+y^{2}+f(z, u)$ for some $f \in C\{z, u\}$.

Proof. By Remark 7, (2), there is at most one even number among $w t s$ of $x, y, z, u$. On the other hand, by Theorem 2 applied to $v=(w t)_{2}$, one has $v(x)+\cdots+v(u)>2+v(\varphi) \geqq 4$. Hence exactly one of $w t$ s of $x, y, z, u$ is congruent to $0 \bmod (2)$. Then Lemma 11 is clear.

THEOREM 12. If $\varphi_{2}$ has rank $\geqq 2$, then after permutation of $x, y, z, u$ (if necessary), one of the following holds.

(1) $w t x+w t y \equiv 0 ; w t z \equiv w t \varphi \equiv 0 ; w t x$, wt $y$, wt $u$ are prime to $m$, and modulo $Z_{m}$-automorphism, $\varphi=x y+f\left(z, u^{m}\right)$ for some $f \in C\left\{z, u^{m}\right\}$.

(2) $m=4$; wts of $x, y, z, u, \varphi$ are 1, 3, 1, $2 \bmod 4$ (after choosing a generator of $\left.Z_{4}\right)$, and modulo $Z_{4}$-automorphism, $\varphi=x^{2}+y^{2}+f\left(z, u^{2}\right)$ for some $f \in C\left\{z, u^{2}\right\}$.

(3) $m=2$; wts of $x, y, z, u, \varphi$ are $1,0,1,1,0 \bmod 2$, and modulo $Z_{2}$-automorphism, $\varphi=x^{2}+y^{2}+f(z, u)$ for some $f \in(z, u)^{4} C\{z, u\}$.

By Lemma 12.2, this follows from Theorem 8, Lemmas 9, 10, 11. (For case (3), one may set $f \in(z, u)^{4} C\{z, u\}$, because otherwise it is reduced to case (1)). 
Remark 12.1. In case (1) (resp. (2), (3)) of Theorem 12, if $f$ is a general linear combination of a finite number of monomials in $\left(z^{2}, u^{m}\right)$ $C\left\{z, u^{m}\right\}$ (resp. $\left(z^{3}, u^{2}\right) C\left\{z^{2}, z u^{2}, u^{4}\right\},\left(z^{4}, z^{3} u, z^{2} u^{2}, z u^{2}, u^{4}\right) C\left\{z^{2}, z u, u^{2}\right\}$ ) and if $f$ has an isolated singular point at 0 , then $(X, p)$ is a terminal singularity. This follows from Corollary 2.1. For example, in case (1), if $v$ is an order, then $v(x)+v(y)+v(z)+v(u)-m-v(\varphi)=(v(x)+v(y)-v(\varphi))+(v(z)-m)$ $+v(u)>0$.

LEMMA 12.2. If $\mathrm{rk} \varphi_{2} \geqq 2$, then modulo permutation of $x, y, z$, $u$ and $\boldsymbol{Z}_{m}$-automorphism, one has either (1) $\varphi_{2}=x y+g(z, u)$ for some quadratic form $g$ in $z$, $u$ or (2) $\varphi_{2} \in k x^{2}+k y^{2}+k z^{2}+k u^{2}$.

Proof. If none of $x^{2}, y^{2}, z^{2}, u^{2}$ appears in $\varphi_{2}$, then one has case (1) by Theorem 8. If for example $x^{2}$ appears in $\varphi_{2}$, then one has $\varphi_{2}=x^{2}+$ $h(y, z, u)$ modulo $Z_{m}$-automorphism for some quadratic form $h$. One can repeat similar argument to $h(y, z, u)$, either to obtain case (1) by applying Theorem 8 , or end up with case (2).

q.e.d.

CoROLlaRy 13. If $\operatorname{rk} \varphi_{2} \geqq 3$, then after permutation of $x, y, z, u$ and choice of generator $\sigma \in Z_{m}$, one has

(1) $\operatorname{rk} \varphi_{2}=4 ; m=2$, wts of $x, y, z, u, \varphi$ are 1, 1, 0, 1, $0 \bmod 2$ and $\varphi=x y+z^{2}+u^{2}$ modulo $Z_{2}$-automorphism,

(2) $\operatorname{rk} \varphi_{2}=3$; either.

(2.1) $m \geqq 2, w t x+w t y \equiv 0, w t z \equiv w t \varphi \equiv 0(m), w t x, w t y, w t u$ are prime to $m$, and $\varphi=x y+z^{2}+u^{i m}$ (im $\geqq 3$ ) modulo $Z_{m}$-automorphism,

(2.2) $m=2$, wts of $x, y, z, u, \varphi$ are $1,1,01,0$, and $\varphi=x y+z^{i}+u^{2}$ $(i \geqq 3)$ modulo $Z_{2}$-automorphism, or

(2.3) $m=4, \sigma$-wts of $x, y, z, u, \varphi$ are $1,1,2,3,2$, and $\varphi=x y+$ $z^{i}+u^{2} \quad\left(i \geqq 3\right.$ : odd) modulo $Z_{4}$-automorphism.

This follows from Theorem 12.

\section{§3. Terminal singularities of type $c D$}

Lemma 14. If $\mathrm{rk} \varphi_{2} \leqq 1$ and $u^{2}$ appears in $\varphi_{2}$, then one has

$$
\varphi=u^{2}+f(x, y, z) \text { modulo } Z_{m} \text {-automorphism, }
$$

where $f \in(x, y, z)^{3} C\{x, y, z\}$ has non-zero cubic term $f_{3}$.

This follows from the definition of $c D V$ points. 
LEMma 15. Under the assumptions and notation of Lemma 14, if $f_{3}$ contains $x y z$ and $m$ is a power of 2 , then $m=2$ and after permutation of $x, y, z$ (if necessary), one has wts of $x, y, z, u, f$ equal to $1,1,0,1,0 \bmod 2$.

Proof. Since $w t x+w t y+w t z \equiv 2 w t u \equiv 0$ (2), at least one of $w t x$, $w t y, w t z$ is even. Without loss of generality, we may assume $w t z$ is even. Then $w t x$, wt $y, w t u$ are odd (Remark 7, (2)). If $m=2$, then Lemma 15 is proved. It remains to disprove the case $m=4$. If $m=4$, then $w t f \equiv 2(4)$ because $w t u$ is odd. Since $f$ contains a power of $z$ (Remark 7, (1)), wt $z \equiv 2$ (4). Choosing $\sigma \in Z_{4}$, one may assume that $\sigma$ $w t s$ of $x, y, z, u$ are $a, b, 2,3(a, b=1,3)$. By $a+b+2 \equiv 2(4)$, one has $a+b \equiv 0$ (4), whence $a+b=4$. Since $w t \varphi \equiv 2$, one sees that the order $v$ induced by this weight satisfies $v(f)=6$. Then $v(x)+v(y)+v(z)+$ $v(u)<4+v(\varphi)$ contradicts Theorem 2 .

q.e.d.

Lemma 16. Under the assumptions and notation of Lemma 14, if $f$. contains $x y z$, then $m$ is a power of 2 .

Proof. Assuming that $m$ is odd, we will derive a contradiction.

(16.1) Claim: $w t x, w t t, w t z$ are prime to $m$.

For example, assume $n=(w t x, m)>1$. Then $w t f \equiv 0(n)$ by Remark 7, (1). Since $n$ is odd, one has $w t u \equiv 0(n)$. This means $n=(w t x, w t u$, $m)>1$, which contradicts Remark 7, (2).

(16.2) Claim: $w t x+w t y+w t z$ and $w t u$ are prime to $m$.

Since $m$ is odd and $w t u^{2} \equiv w t x y z(m)$, it is enough to show (wt $x y z$, $m)=1$. For this, it is enough to derive a contradiction by assuming that $w t x y z \equiv 0(m)$, and $m>1$ is odd. By replacing $w t$ by $-w t$ if necessary, one may assume (by (16.1))

$$
(w t x)_{m}+(w t y)_{m}+(w t z)_{m}=m .
$$

Hence, for the order $v=(w t)_{m}$, one has $v(\varphi)=m$ and $v(x)+v(y)+v(z)+$ $v(u) \leqq m+v(\varphi)$. This is a contradiction to Theorem 2 .

(16.3) By choosing $\rho \in Z_{m}-\{0\}$, one can assume

$$
(\rho-w t x)_{m}+(\rho-w t y)_{m}+(\rho-w t z)_{m} \equiv \pm 1(m) \text {, and }<3 m / 2 \text {. }
$$

Then $(\rho-w t x)_{m}+(\rho-w t y)_{m}+(\rho-w t z)_{m}=m \pm 1$, and for $v=(\rho-w t)_{m}, v(f)=$ $m \pm 1$ is even. By Corollary $2.2,0<m \pm 1-m-(m \pm 1) / 2=(-m \pm 1) / 2$. This is a contradiction, and Lemma 16 is proved. 
Proposition 17. Under the assumptions and notation of Lemma 14, if $f_{3}$ contains $x y z$, then $m=2$, and after permutation of $x, y, z$, one has wts of $x, y, z, u, f$ equal to $1,1,0,1,0 \bmod 2$.

This follows from Lemmas 15, 16.

LEMma 18. Under the assumptions and notation of Lemma 14, if $f_{3}$ contains $x y^{2}$, and if $m>1$ is a power of 2 , then $m=2$ and wts of $x, y$, $z, u, f$ are $0,1,1,1,0 \bmod 2$.

Proof. Assume $m=4$. By $2 w t u=w t x+2 w t y, w t x$ is even whence $w t y$ and $w t u$ are odd (Remark 7, (2)). Thus $2 w t u \equiv 2 w t y$ (4) and $w t x \equiv 0$ (4). Thus by Remark 7, (1), wt $f \equiv 0$ (4), which contradicts $w t u \equiv 1$ (2). The rest is easy.

q.e.d.

LEMMA 19. Under the assumptions and notation of Lemma 14, if $f_{3}$ contains $x y^{2}$, and if $m>1$ is odd, then $m \equiv 3$ and after choosing a generator of $Z_{m}$, one has wts of $x, y, z, u, \varphi$ equal to $2,2,1,0,0 \bmod 3$ and $f_{3}$ contains $x y^{2}, z^{3}$.

Proof. (19.1) Claim: wt $x, w t y, w t z$ are prime to $m$.

It is enough to derive a contradiction from (e.g.) $w t x \equiv 0(m)$. By Remark 7, (1), some power of $x$ appears in $f$, and $w t u \equiv(w t f) / 2 \equiv 0(m)$, which contradicts Remark 7, (2).

(19.2) Claim: By (19.1), we may choose a generator $\sigma$ of $Z_{m}$ so that $\sigma-w t x \equiv 2, \sigma-w t y \equiv b, \sigma-w t z \equiv c(m)$, with $0<b, c<m$. Then, for $v=$ $(\sigma-w t)_{m}, v(f)=2+2 b-m$.

From $v\left(x y^{2}\right)=2+2 b \leqq 2 m$ (19.1), follows $v(f)=2+2 b$ or $2+2 b-m$. But if $v(f)=2+2 b$, then $v(f)$ is even and $2+b+c-m-(2+2 b) / 2=$ $c+1-m \leqq 0$ by (19.1), which contradicts Corollary 2.2.

(19.3) Claim: Under the assumptions and notation of (19.2), if $2 c<m$ and if $m \geqq 5$, then $2 b>m$ and for $w=((2 \sigma)-w t)_{m}$, one has $w(x)=4, w(y)$ $=2 b-m, w(z)=2 c, w(f)=4+4 b-2 m$.

By (19.2), $v(f)=2+2 b-m \geqq 3$, whence $2 b>m$. Thus $w\left(x y^{2}\right)=4+$ $4 b-2 m$. If $w(f) \neq 4+4 b-2 m$, then $w(f)=4+4 b-3 m$ because $b<$ $m$. Then $w(f)$ is odd and $w(x), w(z)$ are even. Thus $w(f)$ is attained by a term $y \cdot$ (monomial of degree $\geqq 2$ ). But

$$
\begin{aligned}
& w(y \cdot(\operatorname{deg} \geqq 2))-(4+4 b-3 m) \\
& \quad \geqq 2 b-m+2-(4+4 b-3 m)=2(m-1-b) \geqq 0,
\end{aligned}
$$


and if the equality holds, then $b=m-1$ and $w((\operatorname{deg} \geqq 2))=2$. This is impossible by $m \geqq 5$. Thus (19.3) is proved.

(19.4) Claim: Under the situation of (19.2), if $m \geqq 5$, then one has $c \geqq(m-1) / 2$.

If $c<(m-1) / 2$, then by (19.3), Corollary 2.2 applied to $w(f)$ gives $0<4+2 b-m+2 c-m-(4+4 b-2 m) / 2=2+2 c-m \leqq 2+(m-2)$ $-m=0$, which is a contradiction. Thus (19.4) is proved.

(19.5) Claim: Under the situation of (19.2), one has (1) if $m \geqq 5$ then $c$ is odd and $c \leqq m-4$, and (2) if $m=3$ then $c=1$ and $b=2$.

Since $v(y \cdot(\operatorname{deg} \geqq 2))-(2+2 b-m)=b+2-(2+2 b-m)=m-$ $b>0$ by $b<m(19.2), v(f)$ is attained by a term containing only $x$ and $z$. Since $v(f)$ is odd, $v(z)=c$ is odd. Since $2+2 b-m \leqq m$ and $3 c>m$ (19.4), $f$ must contain $x z$ if $c \geqq m-2$ and $m \geqq 5$. This is absurd, and hence $c \leqq m-4$. The case $m=3$ is similar.

Let $c=m-2 e, e \geqq 2$. Then $e$ is prime to $m$ by (19.1).

(19.6) Claim: Under the situation of (19.2), there is no integer $i$ such that

$$
m /(2 e-1) \leqq i \leqq 2 m /(2 e+1) .
$$

Assume that such $i$ exists. Then $w^{\prime}=((i \cdot \sigma)-w t)_{m}$ satisfies $w^{\prime}(x)=$ $2 i, w^{\prime}(y)=b^{\prime}, w^{\prime}(z)=2 m-2 i e$, where $b^{\prime}=(b i)_{m}$ satisfies $0<b^{\prime}<m$ by (19.1). We will derive a contradiction to prove (19.6). From $w^{\prime}(f) \equiv 2 i$ $+2 b^{\prime}, 2 i<m$, and $2 b^{\prime}<2 m$, follows that $2 i+2 b^{\prime}<3 m$. Thus $w^{\prime}(f)=$ $2 i+2 b^{\prime}, 2 i+2 b^{\prime}-m$, or $2 i+2 b^{\prime}-2 m$. If $w^{\prime}(f)=2 i+2 b^{\prime}-m$ (odd), then $w^{\prime}(f)$ is attained by $x^{p} y^{q} z^{r}$ with $q>0\left(w^{\prime}(x), w^{\prime}(z)\right.$ are even). Hence

$$
\begin{aligned}
& 2 i+2 b^{\prime}-m=p(2 i)+q b^{\prime}+r(2 m-2 i e), \text { or } \\
& 2 i+b^{\prime}=m+(q-1) b^{\prime}+p \cdot 2 i+r(2 m-2 i e) .
\end{aligned}
$$

Since $2 i \leqq m-1, b^{\prime} \leqq m-1$ (19.1), one has $q=1, p=0$. By $p+q+r$ $\geqq 3$, one gets $r \geqq 2$ and $2 i+b^{\prime}>b^{\prime}+2(2 m-2 i e)$ contradicting (*). If $w^{\prime}(f)=2 i+2 b^{\prime}-2 m$ (even), then it is attained by $x^{p} y^{q} z^{r}, p+q+r \geqq 3$, and

$$
2 i+2 b^{\prime}-2 m=2 i p+b^{\prime} q+r(2 m-2 i e) .
$$

Since $2 i+2 b^{\prime}-2 m<2 i$, one gets $p=0$. Also from $2 i+2 b^{\prime}-2 m<2 b^{\prime}$ $-m<b^{\prime}$, follows $q=0$ and $r \geqq 3$. Hence $2 i>2 i+2 b^{\prime}-2 m=r(2 m-2 i e)$ 
contradicting $(*)$ in (19.6). Thus $w^{\prime}(f)=2 i+2 b^{\prime}$. Now $w^{\prime}(f)$ is even, and Corollary 2.2 applied to $w^{\prime}$ gives a contradiction to $(*)$ :

$$
0<2 i+b^{\prime}+(2 m-2 i e)-m-\left(2 i+2 b^{\prime}\right) / 2=m-(2 e-1) i .
$$

(19.7) Claim: Under the situation of (19.2), if $m \geqq 5$, then one has $e=2, m=7$.

We note $m \geqq 4 e-1$ by (19.4), and hence

$$
\begin{aligned}
& (2 e-1)(2 e+1)\{2 m /(2 e+1)-m /(2 e-1)-1\} \\
& \quad=(2 e-3) m-4 e^{2}+1 \geqq(2 e-3)(4 e-1)-4 e^{2}+1=4 e^{2}-14 e+4 .
\end{aligned}
$$

Thus if $e \geqq 4$, then $2 m /(2 e+1)>m(2 e-1)+1$, and there exists $i$ satisfying $(*)$ in (19.6). Hence $e \leqq 3$. Then it is easy to check (19.7) for $e \leqq 3$ and $m \geqq 5$.

(19.8) Claim: The case $e=2$ and $m=7$ does not give a terminal singularity.

One has $c=3$. By (19.2), $2+2 b-7 \geqq 3$ and $b \geqq 4$, Since $v(x), v(y)$, $v(z) \geqq 2$, one has by (19.2), $2+2 b-7 \geqq 3 \cdot 2$, whence $b=1$. Then for $w^{\prime \prime}=((3 \cdot \sigma)-w t)_{m}, w^{\prime \prime}(x)=6, w^{\prime \prime}(y)=4, w^{\prime \prime}(z)=2$, and $w^{\prime \prime}(f)=14$, whence $w^{\prime \prime}(x)+w^{\prime \prime}(y)+w^{\prime \prime}(z)-7-w^{\prime \prime}(f) / 2=-2<0$, which is a contradiction to Corollary 2.2.

(19.9) Claim: If $m=3$, then $f_{3}$ contains $z^{3}$.

Otherwise, by (19.2) and (19.5), $v(x)=v(y)=2, v(z)=1$, one gets $v(f)=6$, which contradicts (19.2). This proves Lemma 19 .

Proposition 20. Under the situation of Lemma 14, if $f_{3}$ contains $x y^{2}$, then (1) $m=2$ and $w$ ts of $x, y, z, u, f$ are $0,1,1,1,0 \bmod 2$, or (2) $m=3$ and after choosing generator $\sigma$ of $Z_{3}$, one has $\sigma$-wts of $x, y, z, u, f$ equal to $2,2,1,0,0 \bmod 3$, and $f_{3}$ contains $x y^{2}, z^{3}$.

Proof. By Lemmas 18, 19, it remains to exclude $m=6$. By Lemma 19, $f_{3}$ contains $z^{3}$. This implies $3 \cdot w t z \equiv w t f$, which contradicts Lemma 18.

LEMMA 21. Under the situation of Lemma 14, if $f_{3}$ does not contain cross terms (like $\left.x y^{2}, x y z\right)$, then $f_{3} \in C \cdot x^{3}+C \cdot y^{3}+C \cdot z^{3}$.

This is obvious.

LEMma 22. Under the situation of Lemma 21, if $f_{3}=x^{3}+y^{3}+z^{3}$ (resp. $\left.x^{3}+y^{3}\right)$, then $m=3$, and after choosing generator $\sigma$ of $Z_{3}$ and permutation 
of $x, y, z$ (resp. $x, y$ ) if necessary, one has $\sigma$-wts of $x, y, z, u, \varphi$ equal to 1,2 , $2,0,0 \bmod 3$.

Proof. (22.1) Claim: 2 and 3 are the only possible prime factors of $m$.

Assuming that $(m, 6)=1, m>1$, we will derive a contradiction. From $w t x \equiv w t y(m)$, one has $(w t x, m)=1$ by Remark 7 , (2). Thus one can choose a generator $\rho$ of $Z_{m}$ such that $\rho$-wt $x \equiv 2$. Then $\rho-w t$ s of $x, y, z$, $u, \varphi$ are $2,2, c, 3,6$, where $c$ is prime to $m$ by $(w t \varphi, m)=1$, and Remark 7 , (1). Let $v=(\rho-w t)_{m}$. Since $v(\sigma) \geqq 2, m \geqq 5$, one has $v(\varphi)=6$. Thus $v(x)+v(y)+v(z)+v(u)-m-v(\varphi)=c-m+1 \leqq 0$, which contradicts Corollary 2.2.

(22.2) Claim: $(m, 2)=1$.

Indeed if $m=2$, then $w t x \equiv w t y \equiv 0$ (2). This contradicts Remark 7, (2).

(22.3) Claim: $m=3$.

We will derive a contradiction assuming $m=9$. From $2 \cdot w t u \equiv 3 \cdot w t y$ (9), follows $w t u \equiv 0$ (3). By Remark 7, (2), wt x, wt $y, w t z$ are prime to 3 . Thus $w t u \neq \equiv 0$ (9). If $w t x \equiv w t y(9)$, then $Z_{9}$-automorphism: $x \rightarrow x+\alpha y$, keeping $y, z, u$ reduces the problem to Proposition 20, which contradicts $m=9$. Thus $w t x \equiv w t y(3)$, wt $x \neq \equiv w t y(9)$. Choosing a generator $\rho \in Z_{9}$, one may assume $\{\rho-w t x, \rho-w t y\}=\{2 \bmod 9,5 \bmod 9\}$. By exchanging $x, y$ if necessary, one may assume that $\rho$-wts of $x, y, z, u$ are $2,5, c, 3$ $\bmod 9$ ( $c$ is prime to 3 by Remark 7, (2)). By applying Theorem 2 to $v=$ $(\rho-w t)_{9}$, one gets $v(f)=6, c>5$. For $w=((-\rho)-w t)_{9}, w(f) \equiv 3(9)$. By Theorem 2, one gets $w(x)+w(y)+w(z)+w(u)=26-c>9+w(f)$. Thus by $c>5, w(f)=3$. Then $z^{3}$ appears in $f$ and $w(z)=1, c=8$. Thus $(-3 \rho)-w t \mathrm{~s}$ of $x, y, z, u$ are $3,3,3,0 \bmod 9$. Hence for $w^{\prime}=((-3 \rho)-w t)_{9}$, $w^{\prime}(\varphi) \geqq 9$, and $w^{\prime}(x)+w^{\prime}(y)+w^{\prime}(z)+w^{\prime}(u) \leqq 9+w^{\prime}(\varphi)$. This contradicts Theorem 2, and $m=9$ is excluded.

(22.4) Since $w t u \equiv 0$ (3), wt $x$, wt $y$, wt $z$ are prime to 3 . If $w t x \equiv$ $w t y \equiv w t z(3)$, then we can choose $w t$ so that $w t x \equiv 1$. Then setting orders of $x, y, z, u$ equal to $1,1,1,3$, one has an order $v$ such that $v(\varphi)=3$. Then $v(x)+v(y)+v(z)+v(u)=v(\varphi)+3$, which contradicts Theorem 2 . If $f_{3}=x^{3}+y^{3}+z^{3}$, then after permutation of $x, y, z$ and change of weight, one gets $w t$ s of $x, y, z, u, \varphi$ equal to $1,2,2,0,0$. If $f_{3}=x^{3}+y^{3}$, then after permutation of $x, y$ if necessary and change of weight, one gets $w$ ts of $x, y, z, u, \varphi$ equal to $2, b, 1,0,0,(b=1,2) \bmod 3$. If $b=2$, then $2,2,1,3$ 
for $x, y, z, u$ gives order $w$ such that $w(\varphi)=6$ because $\varphi$ does not contain $z^{3}$. Then $w(x)+w(y)+w(z)+w(u)-3-w(\varphi)=-1$, which contradicts Theorem 2. Thus $b=1$. Changing $w t$ to $-w t$, one gets Lemma 22 .

Theorem 23. Under the situation of Lemma 14, if $f_{3}$ is not a cube of $a$ linear factor, then after permutation of $x, y, z$ and choice of generator $\sigma$ of $Z_{m}$, one of the following holds.

(1) $m=2$, wts of $x, y, z, u, \varphi$ are $1,1,0,1,0 \bmod 2$, and $x y z$ or $y^{2} z$ appears in $f_{3}$.

(2) $m=3, \sigma$-wts of $x, y, z, u$, are $1,2,2,0,0 \bmod 3$, and $f_{3}=x^{3}+$ $y^{3}+z^{3}, x^{3}+y z^{2}$, or $x^{3}+y^{3}$ modulo $Z_{3}$-automorphism of $C\{x, y, z\}$.

This follows from Propositions 17, 20, and Lemmas 21, 22.

Remark 23.1. In cases (1), (2) of Theorem 23, it is easy to see that modulo $Z_{m}$-automorphism of $C\{x, y, z, u\}$, one may put $\varphi$ in one of the following forms by Theorem 3:

Case 1. $m=2$, wts of $x, y, z, u$ are $1,1,0,1 \bmod 2$,

$$
\begin{gathered}
\varphi=u^{2}+x y z+x^{2 a}+y^{2 b}+z^{c}, \\
\varphi=u^{2}+y^{2} z+\lambda y x^{2 a-1}+g, \\
\left(a, b \geqq 2, c \geqq 3, \lambda \in k, g \in\left(x^{4}, x^{2} z^{2}, z^{3}\right) C\left\{x^{2}, z\right\}\right),
\end{gathered}
$$

Case 2. $m=3$, wts of $x, y, z, u$ are $1,2,2,0 \bmod 3$,

$$
\begin{gathered}
\varphi=u^{2}+x^{3}+y^{3}+z^{3}, \\
\varphi=u^{2}+x^{3}+y z^{2}+x y^{4} \cdot \lambda+y^{6} \cdot \mu, \\
\varphi=u^{2}+x^{3}+y^{3}+x y z^{3} \cdot \alpha+x z^{4} \cdot \beta+y z^{5} \cdot \gamma+z^{6} \cdot \delta, \\
\left(\lambda, \mu \in C\left\{y^{3}\right\}, 4 \lambda^{3}+27 \mu^{2} \neq 0, \alpha, \beta, \gamma, \delta \in C\left\{z^{3}\right\}\right) .
\end{gathered}
$$

For (23.1.1) and (23.2.1), $(X, p)$ is terminal. (This follows from Corollary 2.1.) If $\varphi$ is a general linear combination of a finite number of monomials as in (23.1.2), (23.2.2), or (23.2.3), and if $\varphi$ has an isolated singularity at 0 , then $(X, p)$ is terminal. (This also follows from Corollary 2.1.)

The way to put $\varphi$ in the standard forms above is as follows. If $m=2$ and $f_{3}$ contains $x y z$, then after operating $Z_{2}$-automorphism, one may assume that $f_{3}=x y z+\lambda z^{3}(\lambda \in k$ ). If $x y h$ (resp. if it is not reduced to (23.1.2) $y z h$, $z x h)$ appears in $f$ for some monomial $h \in(x, u, z)^{2}$, then $Z_{2}$-transformation $z \rightarrow z+\lambda h$ (resp. $x \rightarrow x+\lambda h, y \rightarrow y+\lambda h$ ) kills $x y h$ (resp. $y z h, z x h$ ) for some $\lambda$. Thus for any $n \gg 0$, there is a $Z_{2}$-automorphism $\psi$ such that $\varphi \equiv \psi\left(u^{2}+\right.$ 
$\left.x y z+x^{2 a} \alpha(x)+y^{2 b} \beta(y)+z^{c} \gamma(z)\right)$ modulo $(x, y, z, u)^{n}, \quad(\alpha, \beta$ are even power series; $\alpha(0) \beta(0) \gamma(0) \neq 0 ; \infty \geqq a, b, c ; a, b \geqq 2 ; c \geqq 3)$. Thus, by Theorem 3 , there is a $Z_{2}$-automorphism $\psi$ such that

$$
\varphi=\psi\left(u^{2}+x y z+x^{2 a} \alpha+y^{2 b} \beta+z^{c} \gamma\right) .
$$

Hence one may assume

$$
\varphi=u^{2}+x y z+x^{2 a} \alpha+y^{2 b} \beta+z^{c \gamma} .
$$

Since $\varphi=0$ has an isolated singularity, one sees $a, b, c<\infty$. It is easy to see that there are invariant units $u_{1}, u_{2}, u_{3}, u_{4}$ of $C\{x, y, z\}$ such that

$$
u_{4}^{2}=u_{1} u_{2} u_{3}=u_{1}^{2 a} \alpha=u_{2}^{2 b} \beta=u_{3} \gamma \text {. Then } Z_{2} \text {-automorphism }
$$

$\tau$ of $C\{x, y, z, u\}$ such that $\tau x=u_{1} x, \tau y=u_{2} y, \tau z=u_{3} z, \tau u=u_{4} u$ satisfies

$$
\tau \varphi=u_{4}^{2}\left(u^{2}+x y z+x^{2 a}+y^{2 b}+z^{c}\right) .
$$

Thus (23.1.1) is obtained, and other cases are similar.

\section{§4. Terminal singularities of type $c E$}

Lemma 24. Under the situation of Lemma 14, if $f_{3}=x^{3}$, then modulo $Z_{m}$-automorphism,

$$
\varphi=u^{2}+x^{3}+g(y, z) x+h(y, z),
$$

where $g, h \in C\{y, z\}, g \in(y, z)^{3}, h \in(y, z)^{4}$.

This is obvious.

Theorem 25. Under the situation of Lemma 24, one has $m=2$ and wts of $x, y, z, u, \varphi$ are $0,1,1,1,0 \bmod 2$, and $h \notin(y, z)^{5}$.

Remark 25.1. If $m=2$ and $w t$ s of $x, y, z, u$ are $0,1,1,1 \bmod 2$ and if an even polynomial $g$ (resp. $h$ ) in $y, z$ is a general linear combination of a finite number of monomials $\in(y, z)^{4}$ and $h \notin(y, z)^{5}$, and if

$$
\varphi=u^{2}+x^{3}+g(y, z) x+h(y, z)
$$

has an isolated singularity at 0 , then $(X, p)$ is terminal. (This follows from Corollary 2.1.)

Proof. For an integer $n \geqq 0$, let $g_{n}$ (resp. $h_{n}$ ) be the homogeneous part of degree $n$ of $g$ (resp. $h$ ). We will treat two cases.

Case 1. $m$ is an odd prime. 
In this case, we will derive a contradiction in several steps.

(25.2) Claim: $w t x$, wt $y$, wt $z$ are prime to $m$.

If (e.g.) $w t x \equiv 0(m)$, then by Remark 7, (1), wt $f \equiv 0(m)$ and $w t u \equiv 0$, which contradicts Remark 7, (2).

(25.3) Claim: One may assume that $m$ is a prime number $>7$.

It is enough to derive a contradiction assuming $m \leqq 7$. By (25.2), let $\rho$ be a generator $Z_{m}$ of such that $\rho-w t x \equiv 2(m)$. Since $2 w t u \equiv 3 \cdot w t x$ $(m), v=(\rho-w t)_{m}$ satisfies $v(f)=6$. Let $b=v(y), c=v(z)$. Then by Corollary $2.2,2+b+c-m-3>0$ and $b+c \geqq m+2$. Let $w=((-\rho)-w t)_{m}$. One has $w(x)=m-2, w(y)=m-b, w(z)=m-c$. Since $w\left(x^{3}\right)=3 m-6$, $w(f)=3 m-6$ (odd) or $2 m-6$ (even) because $w(f) \geqq 3$. Thus in any case, by Corollary 2.2, one has $(m-2)+(m-b)+(m-c)>2 m-3$. Hence $m+1>b+c \geqq m+2$. This is a contradiction.

(25.4) Claim: $w t y-w t z$ is prime to $m$.

It is enough to derive a contradiction assuming $w t y \equiv w t z(m)$. Since $\varphi$ defines a $c D V$ point, one of $h_{4}, g_{3}, h_{5}$ is non-zero. If $h_{4} \neq 0$, then after choosing generator $\sigma$ of $Z_{m}$, one has $\sigma$-wts of $x, y, z$ equal to $4,3,3 \bmod m$. Thus Corollary 2.2 applied to the induced order gives $0<4+3+3-m$ $-6=4-m$, which contradicts (25.3). The other cases are the same; if $g_{3} \neq 0$ (resp. $h_{5} \neq 0$ ), then one may assume that the $w t$ s of $x, y, z$ are 3 , 2,2 (resp. 5, 3, 3) $\bmod m$. Hence for the induced order $w, w(f)=9$ (resp. 15 by (25.3)), which contradicts (25.3), by Corollary 2.2 .

(25.5) $g_{3}, h_{4}, h_{5}$ are monomials (or 0). (by (25.3) and (25.4))

(25.6) Claim: None of $g_{3}, h_{4}, h_{5}$ are powers of $y$, or $z$ (up to constants).

By symmetry, enough to show that none of them are powers of $y$. If $h_{4}=y^{4}$ (up to non-zero constants), then one can choose an order $v$ such that $v(x)=4, v(y)=3, v(z)=c(0<c<m)$. Then $v(f)=12$, because $12<$ $m+3$. Then $0<3+4+c-m-6=1+c-m$ (Corollary 2.2), which is a contradiction. If $g_{3}=y^{3}$ (up to non-zero constants), then one can choose an order $v$ such that $v(x)=6, v(y)=4, v(z)=c(0<c<m)$. Then $v(f)=18$ or $18-m$. If $v(f)=18$, then Corollary 2.2 gives a contradiction; $0<6+4+c-m-9=1+c-m$. If $v(f)=18-m$, then $18-m(\leqq 7)$ is a sum of at least 4 numbers which are $4=v(y)$ or $c=v(z)$. Thus $c=1$. Hence one can choose an order $w(-2 w \equiv v(m))$ such that $w(x)=m-3$, $w(y)=m-2, w(z)=(m-1) / 2$. Then $w(f)=3 m-9,2 m-9$, or $m-9$, 
and order of an arbitrary monomial in $x, y, z$ of degree $\geqq 4$ is at least $4 \cdot(m-1) / 2=2 m-2>2 m-9$. Thus $w(f)=3 m-9$ (even). Corollary 2.2 gives $0<(m-3)+(m-2)+(m-1) / 2-m-(3 m-9) / 2=-1$, which is a contradiction. If $h_{5}=y^{5}$ (up to constants), then one can choose an order $v$ such that $v(x)=10, v(y)=6, v(z)=c(0<c<m)$. If $c \geqq 6$, then $v(f)=30(30-m<4.6)$ and Corollary 2.2 gives a contradiction; $0<10+$ $6+c-m-15=c+1-m$. If $c=1,3$, or 5 , then one can choose an order $w(-2 w \equiv v(m))$ such that $w(x)=m-5, w(y)=m-3, w(z)=$ $(m-c) / 2$. For a monomial $M$ in $x, y, z$ of degree $\geqq 4$, one has $w(M) \geqq$ $4 \cdot(m-c) / 2=2 m-2 c>2 m-15$. Thus $w(f)=3 m-15$ (even) by $w\left(x^{3}\right)$ $=3 m-15$. Then Corollary 2.2 gives a contradiction; $0<(m-5)+$ $(m-3)+(m-c) / 2-m-(3 m-15) / 2=-(c+1) / 2$. If $c=2$, or 4 , then one can choose an order $w(2 w \equiv v(m))$ such that $w(x)=5, w(x)=3$, $w(z)=c / 2$. One has $w\left(x^{3}\right)=15$ and $m \geqq 11$ by $(25.3)$, hence $w(f)=15$ (odd) or $15-m$ (even). Then, in any case, Corollary 2.2 gives contradiction; $0<5+3+c / 2-(m+15) / 2<(5-m) / 2$.

(25.7) Claim: One chooses an integer $n \geqq 1$ such that $12 n+5 \geqq m$ $\geqq 12 n-5$. Let $v$ be an order such that $v(x)=4 n, v(y)=b, v(z)=c(0<b$, $c<m$ ). Then $v(f)=12 n$.

Otherwise $v(f)=12 n-m \leqq 5$. Since $v(x) \geqq 4, w(f)$ is attained by $h_{4}$ or $h_{5}$. By (25.4), (25.5), (25.6), $w(f)$ must be attained by $h_{4}, 12 n-m=5$, $(b, c)=(1,2)$ or $(2,1)$. Corollary 2.2 shows $0<4 n+b+c-m / 2-5 / 2=$ $b+c-2 n=3-2 n$. Thus $n=1$ and $m=7$, which is a contradiction to $m>7$.

(25.8) Claim: $b+c>m+2 n$.

This follows from Corollary 2.2 applied to order $v$ in (25.7); $0<4 n$ $+b+c-m-6 n=b+c-m-2 n$.

(25.9) Claim: Let $w$ be an order $(w \equiv-v(m)$, with $v$ in (25.7)) such $w(x)=m-4 n, w(y)=m-b, w(z)=m-c$. Then $w(f)=3 m-12 n$ of $2 m-12 n$.

Otherwise, $w(f)=m-12 n \leqq 5$, because $w\left(x^{3}\right)=3 m-12 n$. Since $w(x)$ $=m-4 n \geqq 12 n-5-4 n=8 n-5 \geqq 3, w(f)$ is attained by $h_{4}$ or $h_{5}$. Since $w(f)$ is odd, $m-12 n=5$, and $(m-b)+(m-c)=3$ by (25.4), (25.6). Let $w^{\prime}$ be an order $\left(w^{\prime} \equiv 3 w(m)\right)$ such that $w^{\prime}(x)=5, w^{\prime}(y)=3(m-b), w^{\prime}(z)$ $=3(m-c)$. Then $w^{\prime}(f)=15$, and Corollary 2.2 shows $0<5+3(m-b)$ $+3(m-c)-m / 2-15 / 2=5+9-m / 2-15 / 2=(13-m) / 2$. Thus $m=11$ 
$=12 n+5$, which is a contradiction.

(25.10) By (25.9), Corollary 2.2, applied to $w$ shows that $0<(m-4 n)$ $+(m-b)+(m-c)-(2 m-6 n)=m+2 n-b-c$. This contradicts (25.8). Thus Case 1 is finished and $m$ is a power of 2 .

Case 2. $m$ is a power of 2.

(25.11) Claim: $w t x \equiv 0$, wt $u$, wt $y, w t z \equiv 1$ (2).

By $2 \cdot w t u \equiv 3 \cdot w t x(2)$, one has $w t x \equiv 0$ (2), and the rest follows from Remark 7, (2).

(25.12) Claim: $g_{3}=h_{5}=0, h_{4} \neq 0$.

Since $w t g_{3} \equiv w t h_{5} \equiv 0(2)$, one has $g_{3}=h_{5}=0$ by (25.11). Since $\varphi$ defines a $c D V$ point, $h_{4} \neq 0$.

(25.13) Claim: $m=2$.

It is enough to derive a contradiction assuming $m=4$. Since $h_{4} \neq 0$, one has $3 \cdot w t x \equiv w t h_{4} \equiv 2 \cdot w t u \equiv 2(4)$, hence $w t x \equiv 2$, wt $y \equiv w t z(4)$. Thus by (25.11), wt $y+w t z \equiv 0$ (4). Let $\rho$ be a generator of $Z_{4}$ such that $\rho$-wts of $x, y, z, u$ are $2, b, c, 3(0<b, c<4)$. Then $b+c=4$. Let $v=$ $(\rho-w t)_{4}$. Then $v(f)=6$, and Theorem 2 gives contradiction; $0<2+b+$ $c+3-4-6=-1$. Thus Case 2 is finished and Theorem 25 is proved.

\section{REFERENCES}

[1] M. Artin, Algebraic approximation of structures over complete local rings, Publication I.H.E.S., no. 36.

[2] M. Artin, Lectures on deformations of singularities, Bombay 1976.

[ 3 ] V. Danilov, Birational geometry of toric 3-folds, Izv. Akad. Nauk SSSR ser. Mat., 46 (1982), no. 5.

[4] D. Morrison and G. Stevens, Terminal quotient singularities in dimensions three and four, preprint.

[5] M. Reid, Canonical 3-folds, Journées de géometrie algébrique d'Angers, ed. A. Beauville, Sijthoff and Noordhoff, Alphen (1980), 273-310.

[6 ] - Minimal models of canonical 3-folds, Advanced studies in pure math. 1, ed. S. Iitaka and H. Morikawa, Kinokuniya and North Holland, 1983, 131-180.

Department of Mathematics

Faculty of Science

Nagoya University

Chikusa-ku, Nagoya 464

Japan 